\title{
Cyclophosphamide Induced Posterior Reversible Encepha- lopathy Syndrome (PRES) in a Patient with Lupus Nephritis
}

\author{
Chandan Buttar, MD, Sofia Lakhdar, MD* (D), Francisco Cuevas Castillo, MD and Adriana Abrudescu (iD
}

Icahn School of Medicine at Mount Sinai/NYC Health + Hospitals Queens, NY, USA

*Corresponding author: Sofia Lakhdar, MD, Department of Medicine, Icahn School of Medicine at Mount Sinai/NYC Health + Hospitals Queens, 82-68 164th Street Jamaica, 11432, New York, USA, Tel: +7865572862

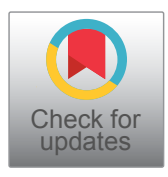

\begin{abstract}
Immunosuppressant agent cyclophosphamide is a therapeutic option for treatment of systemic lupus erythematosus (SLE). Cyclophosphamide use is a predisposing factor to development of posterior reversible encephalopathy syndrome (PRES). PRES is a clinical syndrome of headache, altered mental status, changes in vision and seizures. Precipitating factors for PRES are multifactorial and include cytotoxic medications, changes in blood pressure, metabolic derangements and autoregulatory failure. We report a rare case of cyclophosphamide induced PRES in a patient with SLE. This case highlights the importance of early recognition of this serious adverse effect of cyclophosphamide.
\end{abstract}

\section{Introduction}

Posterior reversible encephalopathy syndrome (PRES) is a clinical pattern of headache, altered mental status, changes in vision, seizures coupled with vasogenic edema involving the parieto-occipital region on neuroimaging [1-4]. PRES is often benign and resolves completely within days to weeks by treatment of the underlying cause and adequate blood pressure control $[5,6]$. Interval radiologic improvement often lags behind clinical recovery. However, death and permanent neurological deficits have been reported. Reoccurrence episodes appear to be infrequent $1<10$ percent) [2]. Precipitating factors are multifactorial and include hypertension, immunosuppressive therapy, renal disease, nephritis. Cyclophosphamide is an alkylating agent widely used in treatment for specific autoimmune and malignant disorder [1]. Reports of cyclophosphamide induced PRES are rare. We report a rare case of cyclophosphamide induced PRES in a patient with SLE.

\section{Case Report}

We present a 22-year-old African American female with a 3-year history of SLE diagnosed in 2017 in Georgia. Patient manifested with polyarthralgia, rash, mouth sores, alopecia and serology positive for SLE. Patient maintained on hydroxychloroquine and remained symptom free until November 2019 when she developed bilateral lower extremity edema and renal failure. Renal biopsy performed showed evidence of stage 4 lupus nephritis and was scheduled to receive 6 cycles of cyclophosphamide. Patient received first dose of cyclophosphamide on 11/26/19. 5 days later patient developed first seizure episode and subsequently developed grand mal status epilepticus requiring mechanical ventilation. MRI performed at the time with a differential diagnosis of PRES vs. lupus cerebritis. Patient received anti-hypertensives, antiepileptics and pulse steroids with interval improvement and eventual discharge from hospital. Patient relocated to NY to continue care and was admitted evaluate need for continuation of dialysis and SLE activity. Patient was given initial pulse steroids $1 \mathrm{~g}$ solumedrol daily for 3 days and maintained on prednisone $30 \mathrm{mg}$ twice daily and hydroxychloroquine $200 \mathrm{mg}$ po daily. Ds-DNA Ab level was 107 (initially > 1400 in Georgia), C3, C4 were normal. Creatinine down-trended from 2.71 to 1.54 and patient did not require hemodialysis. CT head and EEG performed upon admission were unremarkable for acute changes of seizure activity. 


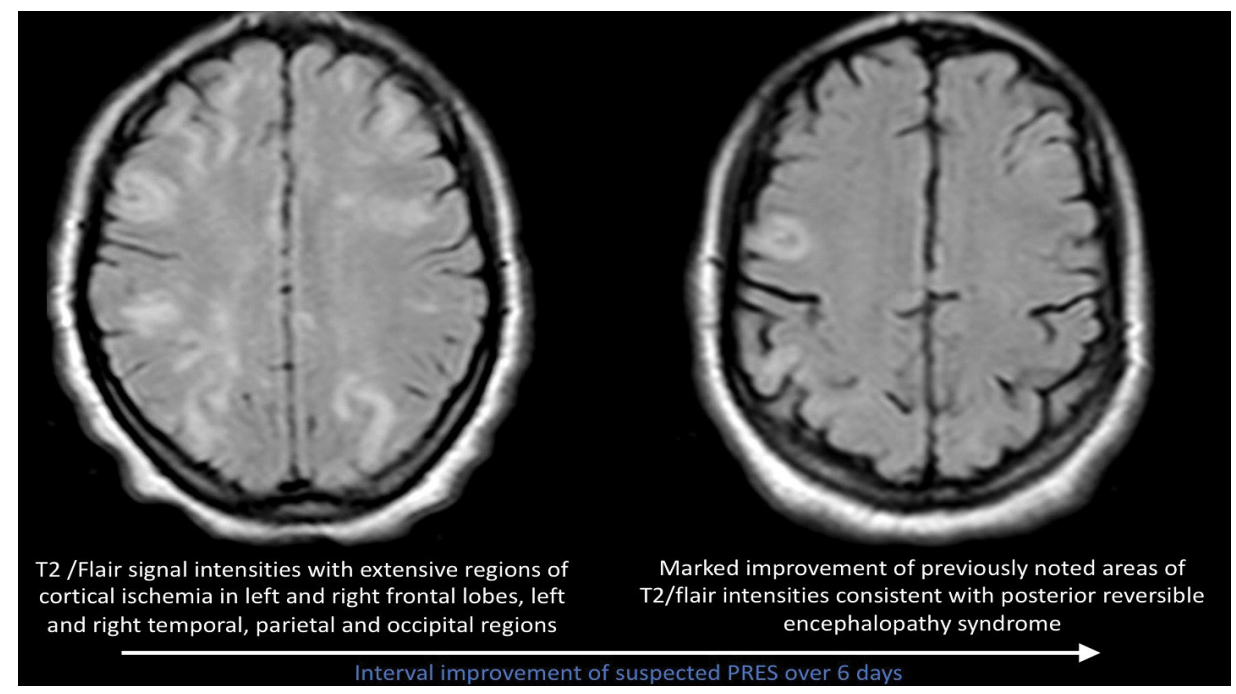

Figure 1: Interval improvement of suspected PRES over 6 days.

Patient received second dose of cyclophosphamide on $1 / 31 / 20$ and two days later patient developed grand mal status epilepticus and severe hypertension requiring mechanical ventilation. MRI of the brain without contrast showed significant changes compared to previous MRI consistent with vasogenic edema within bilateral occipital lobes suggestive of PRES as seen in Figure 1. Patient complained of acute blurry vision, ophthalmological eye exam was normal and the acute blurry vision was likely attributed to cortical vision loss. Patient showed interval improvement of vision and blood pressure. Repeat MRI of the brain 1 week later showed marked improvement in increased T2/flair signal intensities suggestive of resolving PRES.

\section{Discussion}

Our case report highlights a rare and serious complication of cyclophosphamide in patients with lupus nephritis. Risk factors for development of PRES include hypertension, seizure disorder, renal impairment, immunosuppressive treatments including glucocorticoid, cytotoxic agents such as cyclophosphamide $[1,7,8]$. Our patient had active SLE with class IV lupus nephritis for which she received cyclophosphamide, with an initial dose out of state which triggered new onset seizure disorder and hypertension. This was not noted at the time to be secondary to Cyclophosphamide. When the patient presented to our hospital she was maintained on anti-epileptics. Hypertension was controlled. On admission compliments were normal and anti-ds DNA level was trending down and renal function was improving. A few days after receiving second dose of cyclophosphamide patient became acutely ill with status epilepticus and uncontrolled hypertension requiring mechanical ventilation with significant MRI of the brain and EEG changes consistent with PRES.

\section{Conclusion}

Our case report demonstrates occurrence of development of PRES after being challenged with cyclophosphamide on two separate occasions. Physicians should be aware of adverse effects associated with cyclophosphamide use in treatment of SLE nephritis and other autoimmune diseases. Early recognition and appropriate therapy will help ensure a rapid and complete recovery.

\section{References}

1. Jayaweera JL, Withana MR, Dalpatadu CKP, Beligaswatta CD, Rajapakse T, et al. (2014) Cyclophosphamide-induced posterior reversible encephalopathy syndrome (PRES): A case report. J Med Case Rep 8: 442.

2. Tatjana Z, Mirjana SB, Ronald A, Igor A, Srdan N (2017) The multifactorial origin of posterior reversible encephalopathy syndrome in cyclophosphamide-treated lupus patients. Rheumatol Int 37: 2105-2114.

3. Jabrane M, Ait-Lahcen Z, Fadili W, Laouad I (2015) A Case of PRES in an active lupus nephritis patient after treatment of corticosteroid and cyclophosphamide. Rheumatol Int 35: 935-938.

4. Lee $\mathrm{C}-\mathrm{H}$, Lee YM, Ahn SH, Ryu DW, Song JH, et al. (2013) Cyclophosphamide induced posterior reversible encephalopathy syndrome in a patient with lupus nephritis. J Rheum Dis 20: 103-107.

5. Taha N, Abdul RA, Erin F (2019) A rare case of cyclophosphamide-induced posterior reversible encephalopathy syndrome in a patient with anti-GBM vasculitis, and review of current literature. Case Rep Neurol Med 2019: 2418597.

6. Albenz-Abidua MJ, Fuentes B, Diaz D, Royo A, Olea T, et al. (2009) Cyclophosphamide induced reversible posterior leukoencephalopathy syndrome. BMJ Case Reports.

7. Cui H-W, Lei R-Y, Zhang S-G, Han L-S, Zhang B-A (2019) Clinical Features, outcomes and risk factors for posterior reversible encephalopathy syndrome in systemic lupus erythematosus: A case-control study. Lupus 28: 961-969.

8. How J, Blattner M, Fowler S, Wang-Gillam A, Schindler S (2016) Chemotherapy-associated posterior reversible encephalopathy syndrome: A Case Report and Review of the Literature. Neurologist 21: 112-117. 